Preprints of the

Max Planck Institute for

Research on Collective Goods

Bonn 2015/18

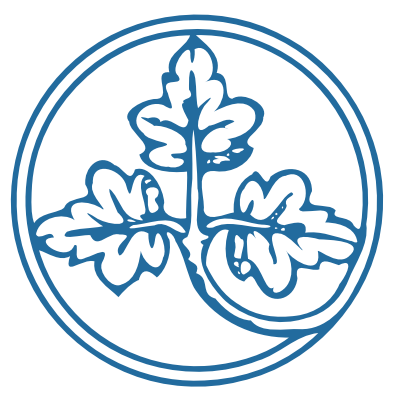

Observing Each Other's

Observations in a Bayesian

Coordination Game

Dominik Grafenhofer

Wolfgang Kuhle

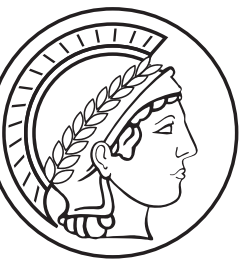




\title{
Observing Each Other's Observations in a Bayesian Coordination Game
}

\author{
Dominik Grafenhofer / Wolfgang Kuhle
}

November 2015

Max Planck Institute for Research on Collective Goods, Kurt-Schumacher-Str. 10, D-53113 Bonn http://www.coll.mpg.de 


\title{
Observing Each Other's Observations in a
}

\section{Bayesian Coordination Game}

\author{
Dominik Grafenhofer, Wolfgang Kuhle ${ }^{1}$
}

\begin{abstract}
We study a Bayesian coordination game where agents receive private information on the game's payoff structure. In addition, agents receive private signals that inform them of each other's private information. We show that once agents possess these different types of information, there exists a coordination game in the evaluation of this information. Even though the precisions of both signal types is exogenous, the precision with which agents forecast each other's actions in equilibrium turns out to be endogenous. As a consequence, there exist multiple equilibria which differ with regard to the way that agents weight their private information to forecast each other's actions. Finally, even though all players' signals are of identical quality, it turns out that efficient equilibria are asymmetric.

Keywords: Coordination Games, Equilibrium Selection, Primary Signals, Secondary Signals
\end{abstract}

This version: November 27, 2015

\section{Introduction}

Games with strategic complementarities give players a strong incentive to choose mutually consistent strategies. In reality such choices are often complicated by the fact that players know neither the game's exact payoffs nor the other player's actions. In such environments player's have to rely on different "pieces" of private information to predict the other player's actions and thus their own payoffs from playing a particular strategy. That is, players try to sense whether and to which extend the other player may be "leaning" towards a particular action or that the other player might "misunderstand" the game, or, respectively, may be under the "wrong impression" as to the situation.

\footnotetext{
${ }^{1}$ Both authors: Max Planck Institute for Research on Collective Goods, Kurt-Schumacher-Str. 10, 53113 Bonn, Germany. We thank Martin Hellwig for comments and for spotting an error. We also received helpful comments and questions from Brian Cooper, Alia Gizatulina, Olga Gorelkina, Alexander Morell, seminar participants in Bonn and Naples, the 2015 Game Theory Conference in Stony Brook, the UECE Lisbon Meetings in Game Theory and Applications 2015, and the EEA conference in Mannheim.
} 
The current paper studies how players use diverse pieces of private information in coordination games. Players receive two types of private information: (i) a "primary signal" that informs them of the game's payoff structure and (ii) a new "secondary signal" that informs players of each other's private beliefs over the games payoff structure. The "secondary" signal models one player's information about the other player's beliefs, biases, optimism, and "wrong impressions" concerning the game. The key prediction of our model is that such heterogeneous pieces of information induce a coordination game in the evaluation of this information. As a consequence, there emerge multiple equilibria which differ in the agents' weighting of different pieces of their private information in order to forecast each other's actions. Even though all players' signals are of identical quality, it turns out that efficient equilibria are asymmetric.

Our model is similar to the ones developed by Rubinstein (1989) and Carlsson and van Damme (1993), where players rely on what we call primary signals to forecast each other's actions. That is, players receive a signal regarding the game's payoffs, which they use to update their beliefs regarding the game's coefficients. Moreover, knowing that the other agent's signal is correlated with their own, they use their signal to infer the other player's posterior beliefs. In the present paper we argue that players often know more than that. Players may observe directly parts of the other player's observation. That is, in the context of the coordinated attack interpretation of the Rubinstein (1989) "electronic mail game", the general may observe that his messenger is off to a "good start". Thus, the chances that he eventually arrives at the other camp are better than usual. The sender of the "primary message" knows that the other player most likely received a message indicating that a particular game was chosen. While he cannot be sure whether it was his messenger or someone else, this observation induces him to revise upward the probability with which the other general received the news. On the other hand, if the sender sees that the messenger is off to a bad start, then he knows that it is less likely that the message will reach its receiver.

The current analysis suggests that such signal environments give rise to two classes of equilibria: (i) symmetric equilibria and (ii) asymmetric equilibria. The distinguishing feature of a symmetric equilibrium will be that agents weight their two signal types equally. In an asymmetric equilibrium one agent leans heavily on the secondary signal, while the other agent has an incentive to lean heavily on his primary signal and vice versa. The key feature is therefore that the two signals are "cross complements". That is, if one player relies heavily on his secondary signal, then the other player has an incentive to rely 
on his primary signal. Such an asymmetric weighting of private signals enables agents to maximize the precision with which they can forecast each other's actions. Finally, to emphasize the importance of the class of asymmetric equilibria, we show that asymmetric equilibria dominate symmetric equilibria on efficiency grounds.

Related Literature: The main contribution of the present paper is the introduction of a new class of private signals. Compared to the literature, we note that Rubinstein (1989), Carlsson and van Damme (1993), and Frankel et al. (2003) have studied two-action coordination games, where agents receive what we call primary signals that allow them to make inference on the game's unknown coefficients. ${ }^{2}$ Moreover, through the correlation of private information agents can reason about each other's posteriors and actions. Regarding equilibrium selection these studies predict that equilibria are symmetric and unique once private signals are sufficiently precise. The present example shows that the existence of secondary private signals can invert this finding: multiple equilibria, symmetric and asymmetric, are ensured once the private signals are sufficiently precise.

Regarding different types of signals, Morris and Shin (2004), Hellwig (2002), Metz (2002), and Angeletos and Werning (2006) emphasize the role of public signals and common priors in the global games framework, showing that such signals restore equilibrium multiplicity if public signals are sufficiently precise compared to the private signal; we give an example where multiplicity arises in pure private signal environments. A further class of signals was introduced by Minelli and Polemarchakis (2003), Angeletos and Werning (2006), and Dasgupta (2007), who study environments where agents observe each other's actions. Such signals tend to induce unique equilibria in the two-player games of Minelli and Polemarchakis (2003), where signals over each other's actions are perfectly revealing. Angeletos and Werning (2006), and Dasgupta (2007) study public signals that partially reveal the other players' actions. They show that multiplicity will emerge if the public signal is of high quality. Kuhle (2015) studies the role of heterogenous priors and gives an example where the public signal's quality reduces the number of equilibria. Rubinstein (1989) points out that equilibrium multiplicity reemerges once there is a technical upper bound for the number of exchanged messages. Similarly, multiplicity also obtains in the model of Binmore and Samuelson (2001), where agents can decide whether or not to send

\footnotetext{
${ }^{2}$ See Frankel et al. (2003) for a broad literature overview on equilibrium selection through what we call primary signals. Carlsson and van Damme (1993), pp. 1008-1010, and Morris and Shin (2007) for a detailed comparison of their "global games", which rely on continuous distributions, with the "electronic mail game" and its discrete information structure.
} 
electronic messages which are costly. The equilibria in the aforementioned models are always symmetric while the current study shows the existence of asymmetric equilibria.

In an alternative interpretation, following Monderer and Samet (1989) and Hellwig (2002), the previously mentioned papers study how different signal structures give rise to different levels of common p-beliefs among players. In this interpretation, we show that the new signal type introduced here allows agents to reach levels of common p-beliefs which are sufficiently high such that they can coordinate on multiple equilibria. That is, the current analysis suggests that coordination games have multiple equilibria if the environment is such that players can observe each other's biases. Moreover, we demonstrate that the way in which players evaluate their information is itself an equilibrium outcome.

The paper is organized as follows. Section 2 outlines our electronic mail game. In Section 2.1, we recall the uniqueness result for the modified game without secondary signals. Section 3 contains the main result. Sections 5 concludes.

\section{A symmetric electronic mail game}

There are two players 1 and 2. Each has two actions $A$ and $B$ to choose from. There is uncertainty about which game $G_{a}$ or $G_{b}$ the two players are going to play. Games $a$ and $b$ differ regarding their payoffs. Nature selects game $a$ with probability $1-p$ and game $b$ with probability $p<\frac{1}{2}$. The game's payoffs are:

\begin{tabular}{|c|c|c|c|c|}
\hline \multicolumn{2}{|r|}{ Game $G_{a}$} & \multicolumn{3}{|c|}{ Game $G_{b}$} \\
\hline & $A \quad B$ & & $A$ & $B$ \\
\hline$A$ & $M, M \quad 0,-L$ & $A$ & 0,0 & $0,-L$ \\
\hline$B$ & $-L, 0 \quad 0,0$ & $B$ & $-L, 0$ & $M, M$ \\
\hline
\end{tabular}

Moreover, we assume $L>M>0$. Hence, players face a coordination problem in both states of the world: if players coordinate on actions $A(B)$ in state $a(b)$, they receive $M$ each, while coordination on $B(A)$ yields 0 to both players. However, if players fail to coordinate, i.e. choose different actions, then the player who plays $B$ receives $-L$, and the payoff for playing $A$ is 0 . Players receive private information on the game's fundamental before they choose an action. The probability $p$, the payoff structure, and the forthcoming communication protocol are common knowledge among players.

Before players choose action $A$ or $B$, they receive information $T_{1}$ and $T_{2}$ respectively: In state $a$, both players get information $T_{1}=T_{2}=0$. In state $b$ one player's computer is randomly selected with probability $\frac{1}{2}$. In turn, this computer sends a message to the other 
player's computer. This message, however, is lost with probability $\varepsilon$. Upon receiving a message this computer sends a confirmation back which is also lost with probability $\varepsilon$. These messages are exchanged until finally one message is lost, and communication ends. Players 1 and 2 now choose their actions based on the number of messages $T_{1}$ and $T_{2}$ that their computers have sent. This means in particular that both players do not know whose computer send the first message when they choose actions. This departure from the original Rubinstein (1989) game makes the game fully symmetric. ${ }^{3}$ In Appendix D we derive our main result for the original asymmetric Rubinstein (1989) game, where Player 1 is informed of the state of nature with probability 1.

\subsection{Rubinstein's Equilibrium}

Before turning to our main findings, we restate the uniqueness result of Rubinstein (1989) for our symmetric mail game.

Proposition 1. There exists only one equilibrium in which player 1 plays $A$ in the state of nature a. In this equilibrium, both players play A, irrespective of the number of sent messages $T_{1}$ and $T_{2}$.

Proof. See Appendix A for the proof via induction.

Proposition 1 recalls the inductive equilibrium selection mechanism that operates through higher-order beliefs: If player 1 plays $A$ for $T_{1}=0$, then player 2 also plays $A$ for $T_{2}=0$, and this induces both players to always play $A$ when $T_{i}>0, i=1,2$. That is, both players continue to play $(A, A)$ despite the fact that their signals inform them that game $b$ was selected, i.e., that playing $(B, B)$ would be payoff-dominant.

Throughout the paper, we study the class of equilibria in which player 1 plays $A$ in the state of nature $a$.

\footnotetext{
${ }^{3}$ That is, the game would be asymmetric if one player knew that he was the first to send a message as is the case in Rubinstein (1989). We make the current assumption to bring out the nature of the asymmetric equilibria more clearly.
} 


\section{Observing each other's observations}

Let us now add a secondary signal $Z_{1}$ and $Z_{2}$ as another source of private information: player $i$ not only gets information $T_{i}$ but also observes

$$
Z_{i}:= \begin{cases}T_{j} & \text { with probability } 1-\psi \\ T_{j}+1 & \text { with probability } \psi .\end{cases}
$$

The secondary signal $Z_{1}$ informs player 1 of the primary signal $T_{2}$ that player 2 received. The secondary signal carries two types of information. First, it allows player 1 to reason about the true fundamental of the game. That is, through its dependence on $T_{2}, Z_{1}$ is correlated with nature's choice of a fundamental. Second, and more importantly, $Z_{1}$ allows player 1 to look more directly at $T_{2}$. This direct look at $T_{2}$ informs him about the probability with which player 2 plays $A$ or $B$. In the following main propositions 3,5 , and 6 , we show that this "direct look" at the other player's signal will induce asymmetric equilibria, in which players weight their signals $Z$ and $T$ differently: if player 1 conditions his actions mainly on his primary signal $T_{1}$, then player 2 will have an incentive to weight signal $Z_{2}$ heavily and vice versa. Put differently, the signals $T_{i}$ and $Z_{j}$ deliver complementary information for the purpose of coordination, while the signals $T_{i}$ and $T_{j}$ do so only to a lesser degree.

To underscore the significance of these asymmetric equilibria, we proceed in three steps. First, we show that they exist. Second, we describe the symmetric equilibria, where agents weight their signals symmetrically. Third, we show that the asymmetric equilibria welfare-dominate symmetric equilibria. Before we study the asymmetric equilibria, we note that the Rubinstein (1989) equilibrium carries over to the environment where agents receive primary and secondary signals.

Proposition 2. When information $T_{1}, Z_{1}$ and $T_{2}, Z_{2}$ are available to players, there exists an equilibrium in which both players play A irrespective of the information received.

Proof. Suppose player 1 thinks that player 2 plays $A$ for sure. Irrespective of $\left(T_{1}, Z_{1}\right)$ the following holds: Choosing $B$ will yield a payoff $-L$, while taking action $A$ will secure him a non-negative payoff. The same argument can be made for player 2 , and thus we have established that the strategy profile $(A, A)$ is an equilibrium.

In this equilibrium, both players receive a zero payoff, even in those situations where they know that playing $(B, B)$ would yield a higher payoff. However, players can use their private signals to coordinate on an alternative class of equilibria: 
Proposition 3. If the secondary signal's precision is sufficiently high, there exist two asymmetric threshold equilibria for every $n \in\{1,2,3, \ldots\}$ : In one equilibrium, player 1 plays $B$ if and only if $T_{1} \geq n+1$, and player 2 plays $B$ if and only if $Z_{2} \geq n+1$ and $T_{2} \geq n$. Reversing the roles of players 1 and 2 yields a second equilibrium.

Proof. Let us consider the first equilibrium with cutoff $n$.

1. Take the behavior of player 2 as given. There are three cases to consider:

(a) $T_{1}<n$ : Player 1 is sure that $Z_{2} \leq n$ and hence plays $A$.

(b) $T_{1}=n$ : With probability $1-\psi$ player 2 's information is $Z_{2}=n$, otherwise (probability $\psi$ ) it is $Z_{2}=n+1$. Playing $A$ secures a payoff of zero for sure; playing $B$ yields an expected payoff smaller than $(1-\psi)(-L)+\psi M$, which is the first player's payoff from $B$, when player 2 always plays $B$ given $Z_{2}>n$. Thus, for $\psi \leq \frac{L}{L+M}=: \psi_{1}$ playing $B$ yields a negative payoff, and hence playing $A$ is optimal.

(c) $T_{1} \geq n+1$ : Player 1 is sure that $Z_{2} \geq n+1$ and $T_{2} \geq n$, hence finds it optimal to play $B$.

2. Equivalently, now take the behavior of player 1 as given.

(a) $Z_{2} \leq n$ : Player 2 knows that $T_{1} \leq n$, and thus plays $A$.

(b) $Z_{2}>n+1$ : Player 2 knows that $T_{1} \geq n+1$, and thus plays $B$.

(c) $Z_{2}=n+1$ : Here we have to take care of four sub-cases:

i. $T_{2}=n-1$ : Hence $T_{1}=n$ for sure and player 2 thus chooses $A$.

ii. $T_{2}=n$ : Defining $\lambda_{\psi}:=P\left(T_{1} \leq n \mid T_{2}=n \wedge Z_{2}=n+1\right)=\frac{\psi}{\psi+\frac{1-\varepsilon}{2}(1-\psi)}$, the payoff for playing $B$ can be written as $\lambda_{\psi}(-L)+\left(1-\lambda_{\psi}\right) M$. From this we obtain a boundary $\psi_{2}:=\frac{(1-\varepsilon) M}{2 L-(1-\varepsilon) M}>0$, which ensures that for all $\psi \leq \psi_{2}$ playing $B$ is optimal for player 2. That is, for $\psi \leq \psi_{2}$ the expected payoff of playing $B$ is non-negative.

iii. $T_{2}=n+1$ : We repeat the same argument using $\mu_{\psi}:=P\left(T_{1} \leq n \mid T_{2}=\right.$ $\left.n+1 \wedge Z_{2}=n+1\right)=\frac{\psi}{\psi+(1-\varepsilon)(1-\psi)}$. It holds that $\mu_{\psi}<\lambda_{\psi}$, such that for all $\psi \leq \psi_{2}$ playing $B$ is optimal for player 2 .

iv. $T_{2}=n+2$ : Hence $T_{1}=n+1$ for sure, and player 2 chooses $B$.

Thus, we can choose $\psi$ sufficiently small, i.e. $\psi \leq \min \left\{\psi_{1}, \psi_{2}\right\}$, such that the strategy profile from the proposition constitutes an equilibrium. 
This shows that secondary signals allow to prevent (to a certain degree depending on $n$ ) infection from players choosing action $A$ when there information is $T_{i}=0$. To interpret the equilibria in Proposition 3 we note that players weight primary and secondary signals asymmetrically. That is, if player 1 switches from playing $A$ to playing $B$ for signals $T_{1} \geq n+1$, then player 2 switches from $A$ to $B$ for signal values $T_{2} \geq n, Z_{2} \geq n+1$. As the proof shows, signals where the trigger strategy requires values greater or equal $n+1$ carry the main information regarding the other player's signals and actions. On the contrary, signals where the trigger strategy requires values greater or equal $n$ carry little information on other player's signals. More precisely, player 1 relies in his inference about the other player's action on the fact that $T_{1} \geq n+1$ informs him of the fact that $T_{2} \geq n, Z_{2} \geq n+1$. Hence, player 1 relies on his primary signal to infer the action of player 2. The main reason for player 1 's reliance on his primary signal $T_{1}$, is that player 2 conditions his actions on $T_{2} \geq n, Z_{2} \geq n+1$. That is, as steps 2.(c) $i-i v$ in the proof show, player 2 relies on his secondary signal to infer the action of player 1 . In turn, player 1 's reliance on the secondary signal $Z_{1}$ justifies player 2 's reliance on the primary signal... This complementarity between player 1's primary and player 2's secondary signal ensures that asymmetric weighting of signals is an equilibrium. Put differently, players face a coordination game in the weighting of their private signals; players can choose their cutoff values for $T_{i}$ and $Z_{i}$ in a way that makes it easy for their counterpart to assess whether their requirement for playing $B$ is met or not. In the present case this means leaning on the primary signal once the opponent leans on the secondary signal and vice versa.

\section{Welfare}

The main purpose of the following propositions 4-6 is to emphasize the role of asymmetric equilibria further. First, we show that there also exist symmetric equilibria, where agents weight their signals equally. Moreover, we show that not every configuration of cutoffs is an equilibrium. Second, proposition 5 underscores that multiple equilibria emerge once private signals are of high quality. Finally, proposition 6 documents that asymmetric equilibria, in which agents exploit the complementarity between primary and secondary signals, welfare dominate the symmetric equilibria of proposition 4.

Proposition 4. If the secondary signal's precision is sufficiently high, there exist symmetric monotone equilibria for every $n \in\{1,2,3, \ldots\}$, where both players play $B$ if and only if $T_{i} \geq n+1$ and $Z_{i} \geq n+1$. There exist no symmetric monotone equilibria, where 
both players play $B$ if and only if $T_{i} \geq n+1$ and $Z_{i} \geq n+2\left(\right.$ or $T_{i} \geq n+2$ and $\left.Z_{i} \geq n+1\right)$.

Proof. See Appendix B.

One might suspect ${ }^{4}$ that the emergence of the different equilibria in Propositions 2-4 depend on the relative precisions of primary and secondary signals, i.e., a high $\varepsilon / \psi$ ratio may be required. This, however, is not the case:

Proposition 5. There exist upper bounds $\bar{\varepsilon}>0$ and $\bar{\psi}>0$, such that the equilibria described in propositions 3 and 4 exist for all combinations of $\varepsilon \leq \bar{\varepsilon}$ and $\psi \leq \bar{\psi}$.

Proof. For propositions 3 and 4 to hold, we need a sufficiently small error probability for the secondary signal, i.e., $\psi \leq \min \left[\psi_{1}, \psi_{2}, \psi_{3}\right]$, where $\psi_{1}=\frac{L}{L+M}, \psi_{2}=\frac{(1-\varepsilon) M}{2 L-(1-\varepsilon) M}$, and $\psi_{3}=\frac{(1-\varepsilon) M}{L+(1-\varepsilon) M}$. It therefore suffices to show that the limits of $\psi_{1}, \psi_{2}, \psi_{3}$ for $\varepsilon \rightarrow 0$ are positive: First, observe that $\psi_{1}$ is positive and does not depend on $\varepsilon$. Second, $\lim _{\varepsilon \rightarrow 0} \psi_{2}=$ $\frac{M}{2 L-M}>0$. Finally, $\lim _{\varepsilon \rightarrow 0} \psi_{3}=\frac{M}{L+M}>0$.

Our results therefore differ from those obtained by Carlsson and van Damme (1993), Frankel et al. (2003), and Morris and Shin (2007), where equilibrium selection works best once private information is very precise. The equilibria in Proposition 3 rely on a coordination game in the evaluation of this information. And this incentive to coordinate is strongest once private signals are very informative.

Finally, we argue that asymmetric equilibria deserve special scrutiny since they are welfare-dominant (in terms of expected total surplus):

Proposition 6. If the secondary signal's precision is sufficiently high, asymmetric equilibria of Proposition 3 welfare-dominate the symmetric ones of Proposition 4 for every given cutoff $n$. Furthermore, the asymmetric equilibria described in Proposition 3 for $n=1$ welfare-dominate those where $n>1$.

Proof. See Appendix C.

That is, once agents exploit the complementarity in weighting the primary and secondary signal, which gives rise to the asymmetric equilibria of proposition 3 , they can anticipate each other's actions with great precision. This increases expected utility since it reduces the probability that, e.g., nature selects game $b$, but players play $(A, A)$, or worse $(A, B)$.

\footnotetext{
${ }^{4}$ In the public and private information frameworks of Hellwig (2002), Morris and Shin (2004), and Angeletos and Werning (2006), multiple symmetric equilibria emerge once public signals or priors are sufficiently precise relative to private signals.
} 


\section{Discussion}

In coordination games, such as the coordinated attack problem, we often have a sense as to the beliefs that the other player may hold on the game that he is involved in. That is, one player may sense that the other player is an "optimist" with regard to a certain action or, similarly, that the other player likely holds a particular "mistaken view" of the situation. The current model incorporates such aspects by giving players noisy information over each other's information. The analysis of such an information structure shows that players who posses different pieces of information face a coordination game as to how they use their private signals to forecast each other's actions. This coordination game in the evaluation of information has multiple symmetric and asymmetric equilibria. Comparison of these equilibria shows that asymmetric equilibria, where players exploit the complementarity between primary and secondary signals, welfare dominate symmetric ones. 


\section{References}

Angeletos, G.-M. and Werning, I. (2006). Crises and prices: Information aggregation, multiplicity, and volatility. American Economic Review, 96(5):1720-1736.

Binmore, K. and Samuelson, L. (2001). Coordinated action in the electronic mail game. Games and Economic Behavior, 35:6-30.

Carlsson, H. and van Damme, E. (1993). Global games and equilibrium selection. Econometrica, 61:989-1018.

Dasgupta, A. (2007). Coordination and delay in global games. Journal of Economic Theory, 134(1):195-225.

Frankel, D., Morris, S., and Pauzner, A. (2003). Equilibrium selection in global games with strategic complementarities. Journal of Economic Theory, 102(1):1-44.

Hellwig, C. (2002). Public information, private information, and the multiplicity of equilibria in coordination games. Journal of Economic Theory, 107:191-222.

Kuhle, W. (2015). A global game with heterogenous priors. Economic Theory Bulletin Forthcoming, pages 1-19.

Metz, C. (2002). Public and private information in self-fulfilling currency crises. Journal of Economics, 76:65-85.

Minelli, E. and Polemarchakis, H. (2003). Information at equilibrium. Economic Theory, 21(2-3):573-584.

Monderer, D. and Samet, D. (1989). Approximating common knowledge with common beliefs. Games and Economic Behavior, 1:170-190.

Morris, S. and Shin, H. S. (2004). Coordination risk and the price of debt. European Economic Review, 48(1):133-153.

Morris, S. and Shin, H. S. (2007). Common belief foundations of global games. Workingpaper, pages 1-49.

Rubinstein, A. (1989). The electronic mail game: Strategic behaviour under 'almost common knowledge'. American Economic Review, 79(3):385-391. 


\section{A Proof of Proposition 1}

The proof is parallel to the one in Rubinstein (1989). First, we establish that player $i$ plays $A$ when $T_{i}=0$. Player $i$ considers two possible scenarios:

1. With probability $(1-p)$, game $G_{a}$ is played.

2. With probability $\frac{1}{2} p \varepsilon$, Player $j$ was selected, game $G_{b}$ is played, and the message from player $j$ to player $i$ was lost.

Hence, we find a lower bound $\tilde{A}$ for i's payoff from playing $A$ and an upper bound $\tilde{B}$ for i's payoff from playing $B$ :

$$
\pi(A) \geq \frac{(1-p) M+\frac{1}{2} p \varepsilon 0}{(1-p)+\frac{1}{2} p \varepsilon}=: \tilde{A} \quad \pi(B) \leq \frac{-(1-p) L+\frac{1}{2} p \varepsilon M}{(1-p)+\frac{1}{2} p \varepsilon}=: \tilde{B}
$$

It holds that $\tilde{A}>\tilde{B}$, and thus player $i$ plays $A$. The induction step from $t-1$ to $t$ is identical to the original Rubinstein one: assume that both players play $A$ when they receive a $T_{i}<t$. Consider that player $i$ gets information $T_{i}=t$. For the following argument we denote the probability that player $i$ was informed first that game $G_{b}$ is played by $\kappa_{t} \in[0,1]$. The posterior probability of player $j$ having received information $T_{j}=t-1$ is given by

$$
z_{t}:=\frac{\kappa_{t} \varepsilon+1-\kappa_{t}}{\kappa_{t}(\varepsilon+(1-\varepsilon) \varepsilon)+1-\kappa_{t}}>\frac{1}{2} .
$$

In other words, the posterior probability of player $j$ playing $A$ is larger than $\frac{1}{2}$, and thus playing $A$ is optimal for player $i$ as well: Playing $A$ yields 0 , while playing $B$ has expected payoff $z_{t}(-L)+\left(1-z_{t}\right) M<0$.

\section{B Proof of Proposition 4}

We start by proving the first statement. Without loss of generality, we have to check only if player $i$ 's best response to player $j$ 's equilibrium strategy is consistent with player $i$ 's equilibrium strategy. We have to check the following cases of information that player $i$ might receive:

1. $Z_{i} \leq n$ : Player $i$ knows that $T_{j} \leq Z_{i} \leq n$ and that $j$ player A, hence plays $A$.

2. $Z_{i}=n+1$ :

(a) $T_{i}=n$ : player $i$ knows that $Z_{j}=n$ and that player $j$ plays $A$, which leads $j$ to play $A$ as well. 
(b) $T_{i}=n+1$ : Clearly $Z_{j} \geq n+1$ and $T_{j} \in\{n, n+1\}$. To determine the payoff of playing $B$, the conditional distribution of $T_{j}$ has to be taken into account:

$$
\frac{(1-\varepsilon)^{2 n} \varepsilon \psi}{(1-\varepsilon)^{2 n} \varepsilon \psi+(1-\varepsilon)^{2 n+1} \varepsilon(1-\psi)}(-L)+\frac{(1-\varepsilon)^{2 n+1} \varepsilon(1-\psi)}{(1-\varepsilon)^{2 n} \varepsilon \psi+(1-\varepsilon)^{2 n+1} \varepsilon(1-\psi)} M .
$$

Hence playing $B$ is optimal if $\psi<\psi_{3}:=\frac{(1-\varepsilon) M}{L+(1-\varepsilon) M}$.

(c) $T_{i}>n+1$ : thus $Z_{j} \geq n+2$ and $T_{j} \geq n+2$. Player $j$ plays $B$ and the optimal response of $i$ is $B$.

3. $Z_{i}=n+2$ : Player $i$ knows that $T_{j} \geq n+1$.

(a) $Z_{i}=n+2$ implies that $T_{i}<n$ is not feasible.

(b) $T_{i}=n: Z_{j} \geq n+1$ with probability $\psi$, hence the payoff of playing $B$ is $(1-\psi)(-L)+\psi M$, which is negative for $\psi<\psi_{1}$, the case when playing $A$ is optimal for player $i$.

(c) $T_{i}>n$ : Therefore $Z_{j} \geq n+1$ for sure, and thus both players play $B$.

4. $Z_{i}>n+2$ : Player $i$ knows that $T_{j} \geq Z_{i}-1>n+1$. It also holds that $T_{i} \geq n+1$ and thus $Z_{j} \geq n+1$. Therefore player $j$ plays $B$ and player $i$ 's best response is to play $B$ as well.

Hence we have established the first part of the proposition for $\psi \leq \min \left\{\psi_{1}, \psi_{3}\right\}$.

To prove the second part of the proposition we provide a counter example: Suppose $Z_{1}=n+1$ and $T_{1}=n+2$ : player 1 now plays $A$. This is not a best response since player 1 knows that $T_{2} \geq n+1$ and $Z_{2} \geq n+2$, and thus that 2 plays $B$ with certainty. Hence, equilibria where players play $B$ iff $T_{i} \geq n+1$ and $Z_{i} \geq n+2$ cannot exist.

\section{Proof of Proposition 6}

We prove the second part of the statement first. That is, we compute the total welfare loss in the asymmetric equilibria of Proposition 3 given $n$ (sum of expected surplus losses of player 1 and 2) compared to hypothetical perfect coordination between both players. Note that in state $a$ neither miscoordination nor coordination on the wrong action can occur. In state $b$

1. coordination on the wrong action $(A, A)$ happens with probability

$$
p\left[1-(1-\varepsilon)^{2(n-1)}+(1-\varepsilon)^{2(n-1)} \varepsilon(1+(1-\psi)(1-\varepsilon))+\frac{1}{2}(1-\varepsilon)^{2 n}(1-\psi)\right]
$$


2. miscoordination $(B, A)$ happens when $T_{1}=n, T_{2} \geq n$, and $Z_{2}=n+1$. The associated probability is $p(1-\varepsilon)^{2 n-1} \varepsilon \psi$.

Using this, we can compute the welfare loss in equilibrium:

$$
\begin{aligned}
& l_{n}:=p\left\{(1-\varepsilon)^{2 n-1} \varepsilon \psi(2 M+L)\right. \\
& \left.+\left[1-(1-\varepsilon)^{2(n-1)}+(1-\varepsilon)^{2(n-1)} \varepsilon(1+(1-\psi)(1-\varepsilon))+\frac{1}{2}(1-\varepsilon)^{2 n}(1-\psi)\right] 2 M\right\} \\
& =p(1-\varepsilon)^{2 n-1}\left\{\varepsilon \psi(2 M+L)+\left[-1+\varepsilon(1+(1-\psi)(1-\varepsilon))+\frac{1}{2}(1-\varepsilon)^{2}(1-\psi)\right] 2 M\right\}+p 2 M .
\end{aligned}
$$

It is straightforward to see that the expression in curly brackets is negative for small $\psi$. Hence, $l_{n}$ is increasing in $n$ for small $\psi$.

The proof of the first part of the proposition requires computing the welfare loss in the symmetric equilibria of Proposition 3 given $n$. Again there are two types of losses:

1. coordination on the wrong action $(A, A)$ happens with probability

$$
p\left[1-(1-\varepsilon)^{2 n}+(1-\varepsilon)^{2 n} \varepsilon(1-\psi)\right]
$$

2. miscoordination $(B, A)$ happens once $T_{1}=n, T_{2} \geq n$, and $Z_{2}=n+1$. Hence it happens with probability $p(1-\varepsilon)^{2(n-1)} \varepsilon \psi$.

Using these probabilities, we compute the welfare loss in equilibrium:

$$
\tilde{l}_{n}=p(1-\varepsilon)^{2(n-1)}\left\{\varepsilon \psi(2 M+L)+(1-\varepsilon)^{2}[-1+\varepsilon(1-\psi)] 2 M\right\}+p 2 M
$$

Note that $l_{n}-\tilde{l}_{n} \underset{\psi \rightarrow 0}{\longrightarrow}-p(1-\varepsilon)^{2 n} \varepsilon M<0$. Hence, for a small enough $\psi$ it holds that $l_{n}<\tilde{l}_{n}$, i.e., welfare is higher in the asymmetric equilibria for every given $n$. 


\section{Referee appendix}

In this appendix we show that our main result, multiplicity of equilibria in the presence of primary and secondary signals, holds for the original asymmetric version of the electronic mail game of Rubinstein (1989). That is, we now assume that it is always player 1 who gets informed in case nature draws game $b$. This is the special case where we set the probability $P=1$ (rather than $1 / 2$, which is what we assumed in the main text). Other than that we leave the signals $Z, T$ unchanged. Our only deviation from Rubinstein (1989) is therefore the introduction of the secondary signal $Z$. Naturally, Proposition 2 holds without modification of the proof.

We now show for this simplified setting that multiple equilibria exist as in the main text. In particular, we prove that the asymmetric equilibria described in Proposition 3 still exist:

Proposition 7. For small enough $\psi$ there exists an asymmetric threshold equilibrium for every $n \in\{1,2,3, \ldots\}$ : player 1 plays $B$ if and only if $T_{1} \geq n+1$ (which implies $Z_{1} \geq n$ ) and player 2 plays $B$ if and only if $Z_{2} \geq n+1$ and $T_{2} \geq n$.

Proof. The proof is mostly unchanged compared to the proof of Proposition 3. There are two exceptions:

2. Equivalently, now take the behavior of player 1 as given.

(c) $Z_{2}=n+1$ : Here we have to take care of four subcases:

ii. $T_{2}=n$ : Note that $P\left(T_{1}=n \mid T_{2}=n \wedge Z_{2}=n+1\right)=\psi$, and thus, the payoff of playing $B$ is given by $\psi(-L)+(1-\psi) M$. From this we can determine $\bar{\psi}_{2}:=\frac{M}{L+M}>0$ such that for all $\psi \leq \bar{\psi}_{2}$ playing $B$ is optimal for player 2, i.e. where the expected payoff of playing $B$ is non-negative.

iii. $T_{2}>n$ : Hence $T_{1} \geq n+1$ for sure, player 2 chooses $B$.

Again, we can choose a small enough $\psi$, i.e., $\psi \leq \min \left\{\psi_{1}, \bar{\psi}_{2}\right\}$, such that the strategy profile from the proposition is indeed an equilibrium. 